\title{
SELF-BUNCHING ELECTRON GUNS
}

\author{
L. K. Len and Frederick M. Mako \\ FM Technologies, Inc., 10529-B Braddock Road, Fairfax, VA 22032
}

\begin{abstract}
This paper presents several new approaches for producing bunched electron beams. The need for higher power injectors and microwave sources for various applications has in turn created the need for higher emission current density electron guns with a means for bunching at the gun. Four different cathode/gun approaches are presented. The first three utilize external methods of bunching. They are based on: field emission, photoemission and ferroelectric emission. The fourth is based on secondary emission and utilizes self-bunching. The latter is emphasized in this presentation. This self-bunching electron gun produces naturally bunched electron beams with the use of microwave fields to generate secondary emission and multipacting of electrons. This type of electron gun is called the Micro-Pulse Gun (MPG). One wall of an RF cavity is made opaque to an input RF electric field but is partially transparent to electrons in order to extract the beam. FMT has studied using simulation codes the resonant bunching process which gives rise to high current densities $(0.01-5$ $\mathrm{kA} / \mathrm{cm}^{2}$ ), high charge bunches (up to $500 \mathrm{nC} /$ bunch), and short pulses (1-100 ps) for frequencies from 1 to $12 \mathrm{GHz}$. The beam pulse width is nominally $~ 5 \%$ of the RF period. Experimental verification of self-bunching, cold electron emission, long life, and tolerance to contamination has been achieved. Measurements of current density, bunch length and lifetime are presented for the MPG.
\end{abstract}

\section{INTRODUCTION}

The creation of high-current, short-duration pulses of electrons has been a challenging problem for many years. High-current pulses are widely used in injector systems for electron accelerators, both for industrial linacs as well as high-energy accelerators for linear colliders. Shortduration pulses are also used for microwave generation, in klystrons and related devices, for research on advanced methods of particle acceleration, and for injectors used for free-electron laser (FEL) drivers [1,2]. Future linear colliders based on two-beam accelerator schemes $[3,4]$ may require individual RF sources capable of 50-75 MW of $\mathrm{RF}$ power with a corresponding pulse width of $0.15-0.3$ $\mu$ s at a frequency of up to $30 \mathrm{GHz}$ and at repetition rates of $60-180 \mathrm{~Hz}$. Because the cost of the RF sources will be a large fraction of the cost of the accelerator, there is a

\footnotetext{
"Work supported by the U. S. Department of Energy under SBIR

\#Email: fmako@erols.com

${ }^{+}$Patents Pending
}

need for high-power microwave sources capable of reliable multi-megawatt performance at high efficiency.

Recent cathode advances have been driven by the need for ultra-high-power devices. These devices require higher emission current densities than thermionic cathodes provide and could benefit from direct modulation of the electron beam at the gun. Direct modulation of the emitted beam can improve the gain and efficiency and reduce the size of microwave amplifiers [5]. In addition direct modulation of the beam to produce a train of short pulses is of interest for use as a buncher for injection into RF linacs. Current density and direct modulation are addressed in the following sections by four different cathode/gun schemes.

\section{FIELD EMISSION}

In this section we present recent developments in field emission and direct modulation of electron beams using field-emission arrays (FEAs). Field-emission cathodes have been fabricated using a variety of materials and methods. Single tips with a radius of curvature about 60 $\mathrm{nm}$ to $90 \mathrm{~nm}$ have been used to generate current densities of more than $10^{8} \mathrm{~A} / \mathrm{cm}^{2}[6,7]$. Arrays of tips have generated current densities of $2.5-40 \mathrm{~A} / \mathrm{cm}^{2}$ (DC) to 2,0002,400 $\mathrm{A} / \mathrm{cm}^{2}$ (pulsed) [8-10], which exceed current densities available from thermionic cathodes. Emission from gated FEAs can be initiated by the application of gate voltages of less than 100 Volts. Among the advantages envisioned for FEAs are the ability to address and control the emission spatially and to directly modulate emission current. Beam emittance from single tips is estimated to be much smaller than any other type of cathode. Normalized beam brightness from single tips with total currents of $10 \mu \mathrm{A}$ is estimated to be on the order of $10^{15}$ $\mathrm{A} /\left(\mathrm{m}^{2}\right.$-steradian) $[11,12]$, or about two to three orders of magnitude better than thermionic cathodes. The emittance from arrays of tips is, however, poor without integrated lenses on each tip [13-16].

NEC Corporation demonstrated two miniaturized TWTs using: lateral resistor-stabilized Spindt-type FEAs [17] and arc-resistant Spindt-type FEAs [18,19]. The arcresistant FEA type TWT achieved 5,000 hours of operation and was terminated by a cause other than arc failure. The electron beam in the TWT is modulated in the conventional manner (not gate modulated). Gate modulation is complicated by the large input capacitance caused by the small distance between the gate and the substrate. A large transconductance, defined as the ratio of the change in emitted current to the change in gate voltage, will help alleviate this problem. Several groups have demonstrated 
emission modulation on top of a DC beam current at frequencies of up to $10 \mathrm{GHz}$ [8-10, 20-26]. This device offers a compact and potentially highly efficient microwave source.

\section{PHOTOEMISSION}

During recent years, considerable effort has been applied to the development of laser-initiated photocathode injectors [27-34]. The best photocathodes have achieved ten's of nanocoulombs per microbunch with a train limited by the available laser systems to contain less than one hundred bunches [34]. Photoemitters have been used mainly until recently as sources of prebunched electron beams in RF linacs. In a typical photoinjector, the photocathode forms an integral part of the first RF cavity before the main linac structure. The photoelectrons from the cathode are quickly accelerated in field gradients of 50-100 $\mathrm{MV} / \mathrm{m}$ so that the electrons reach relativistic velocities within a centimeter from the cathode surface. Thus, debunching effects due to space charge are minimized. Current interest for photocathode injectors is in high power RF generation. Potential applications include devices such as two-beam accelerators $[3,4]$ which have been proposed to generate tens of megawatts at frequencies of 11.4-34.2 GHz. RF power generation relies on the laser to generate a bunch train to form the electron beam. With photocathodes, the charge, bunch length, spatial and temporal distribution of the electrons could be controlled by varying the laser energy, its pulse duration and its spatial and temporal profile. The CLIC facility [34] at CERN combines both injector and RF source aspects of bunched electron emission in its two-beam accelerator. In this device the drive beam consists of 24 bunches of electrons each with $\sim 3 \mathrm{nC}$ charge, $\sim 1.5 \mathrm{~mm}$ bunch length and bunch repetition rate of $3 \mathrm{GHz}$. The prebunched, pre-accelerated beam was decelerated in a slow wave structure to produce $60 \mathrm{MW}$ of RF power at $30 \mathrm{GHz}$ in a $\sim 15 \mathrm{~ns}$ pulse.

\section{FERROELECTRIC EMISSION}

Ferroelectric emission falls broadly into two categories. The first involves a phase transition from ferroelectric to antiferroelectric state [35,36]. The second involves rapid changes in the ferroelectric state [37-42] but does not involve a phase transition. The former can be described by considering the free charge that exists on the surface of a pre-poled ferroelectric cathode. This free charge screens the extremely large electrostatic field from the surrounding electron gun vacuum due to the material polarization. When a rapidly changing electric field is applied across the ferroelectric material, it modifies the amount of surface charge required to produce the electrostatic screening. The resulting surplus charge is ejected into the gun and is available for the production of pulsed electron beams. In the second mode of emission, depending on the polarity of the trigger pulse used to initiate emission it is also possible to generate the electron beam from the metallic grid on the front surface of the ferroelectric. In this configuration the ceramic acts as a high dielectric constant ( $\varepsilon_{r} \sim 1000$ ), which serves to enhance the field causing the emission at the triple points on the front surface of the ferroelectric ceramic. In this mode of use the emission is similar to that found in FEA arrays, where the application of the emission trigger pulse to the gate electrode in the FEA is replaced by pulsing of the rear surface of the ferroelectric emitter. Unlike the previous two emission schemes, relatively little work has been done to date on beam modulation, and work has focused on the understanding of the physics of the emission process. Experiments have been carried out by changing the material (e.g. PLZT) from an antiferroelectric to a ferroelectric state $[35,36]$, and in other cases by cycling a ferroelectric material (e.g. PZT) around a minor hysteresis loop [37-42]. Beam currents up to $350 \mathrm{~A}$ and electron energies in the range of 200-550 keV with pulses in excess of $200 \mathrm{~ns}$ have been achieved [43]. The ferroelectric emission mechanism can easily generate electron beam currents which are suitable for ultra-high-power microwave sources.

\section{SECONDARY EMISSION GUNS}

The electron guns to be described below are based on a natural bunching process which eliminates the need for bunching section(s), timing system, and laser. In this method, short ( 10 ps) high-current pulses are formed by the resonant amplification of an electron current utilizing multipacting from the walls of a microwave cavity. One narrow bunch is transmitted every RF period. This cavitytype bunching electron gun is called the Micro-Pulse Electron Gun or MPG [44]. The MPG is capable of generating high-current and short bunches with low emittance as required for many accelerators and microwave devices. Studies of the multipacting process [45-47] have concentrated on the theoretical aspects of the phenomenon and the RF driver interaction. Bunch stability and its effect on current saturation are treated in reference [48]. References [44] and [49-51] discuss the saturation process, peak currents, and frequency scaling characteristics.

Micropulses are produced by resonantly amplifying a current of secondary electrons in an RF cavity operating in, for example, a $\mathrm{TM}_{010}$ mode (see Fig. 1). Bunching occurs rapidly and is followed by saturation of the current density in ten to fifteen RF periods. Bunching occurs automatically by a natural phase selection of resonant particles. Localized secondary emission in the MPG is determined by material selection. The reason for the name "Micro-Pulse Gun" is the fact that the pulse width is only a few percent of the RF period, in contrast to usual RF guns where it is equal to half the RF period. 


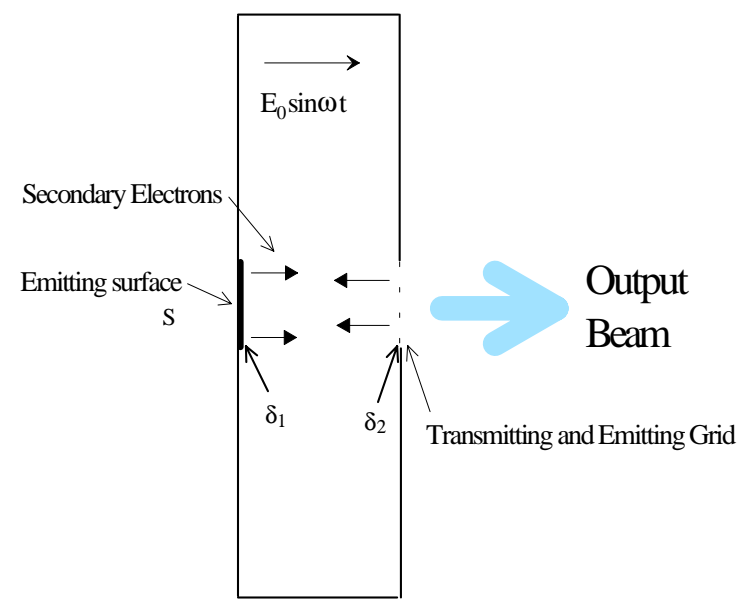

Figure 1: MPG concept for generation of bunched beams. Side view of MPG cavity showing emitting and transmitting grid surfaces. Figure is not to scale.

Radial space charge expansion in the MPG cavity is controlled by a combination of electric and magnetic focusing. The grid not only allows transmission of bunches but also provides an emitting surface for electron multiplication. Emittance growth from the grid in the MPG can be very small due to a unique feature of the MPG. Briefly, the resonant particles are loaded into the wave at low phase angles. They reach the opposite grid $180^{\circ}$ later, and thus exit at low phase angles, thereby experiencing a reduced transverse kick from the grid wires. This minimizes the emittance growth from the grid in the MPG.

\subsection{Experimental Demonstration of the $M P G$}

Figure 2 shows the macropulse operation of an L-band MPG experiment. The L-band MPG cavity is designed for a $\mathrm{TE}_{101}$ mode and is powered by an L-band $(\sim 1.3 \mathrm{GHz})$ magnetron. The magnetron is operated at $300 \mathrm{~Hz}$ repetition rate. Each microwave pulse lasts for $5 \mu$ s (at the base) and contains about 6,313 electron bunches ( 1 for each RF period). The top trace is the RF power in the cavity and the bottom trace is the transmitted macropulse beam current from the MPG on a $1 \mu \mathrm{s} / \mathrm{div}$. time scale. The RF power delivered to the beam is about $50 \mathrm{~kW}$ and the beam current is about $2 \mathrm{~A}$ or about $2 \mathrm{~A} / \mathrm{cm}^{2}$ in the macropulse. The current and current density are not limited to the above values but were the design values for this particular experiment. Note the clean current trace over the full RF pulse length.

When a fast $(50 \mathrm{GHz})$ sampling oscilloscope is used and a $5 \mathrm{~ns}$ slice of the macropulse as indicated in Figure 2 is examined, the micropulses can be observed. Figure 3 shows a measurement of the bunches on a $500 \mathrm{ps} / \mathrm{div}$. time scale. The bunches appear with the periodicity of the RF field ( 792 ps), in excellent agreement with simulation $[44,49,50]$. The pulse amplitude appears to decrease slightly in time because of the location of the $5 \mathrm{~ns}$ slice in the macropulse. More detailed measurements show that the actual bunch length is about 40 ps (FWHM) which is about $5 \%$ of the RF period. In Figure 3 the micropulse current density is about $22 \mathrm{~A} / \mathrm{cm}^{2}$ and is in good agreement with simulation. For this result there is about $1.1 \mathrm{nC}$ or $\sim 7 \times 10^{9}$ electrons per RF period. Lifetime testing has been carried out for about 18 months at almost 24 hours per day with a repetition rate of $300 \mathrm{~Hz}$ and $5 \mu$ s macropulses. During that time, approximately $5.8 \times 10^{13}$ microbunches or 62,000 coulombs $\left(82,000 \mathrm{C} / \mathrm{cm}^{2}\right)$ have passed through this gun and it is still working flawlessly.

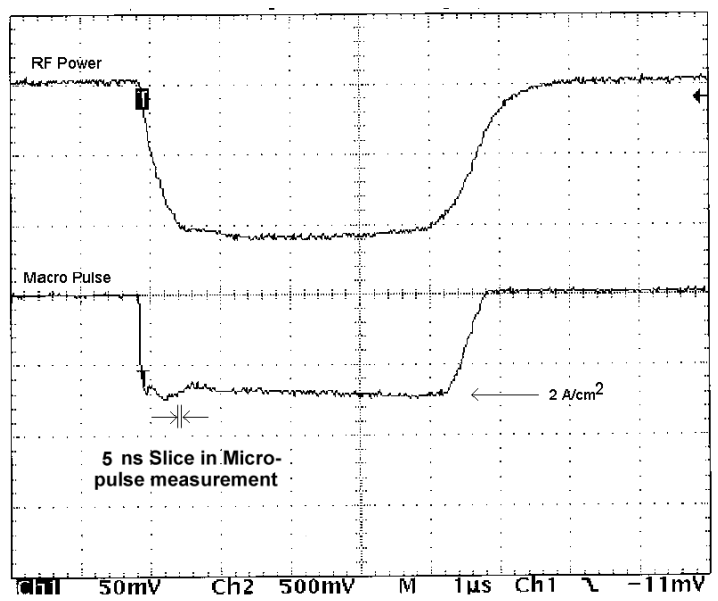

Figure 2: Oscilloscope traces of macropulse operation of the L-band experiment. Top trace: the RF power in the cavity. Bottom trace: the transmitted beam current.

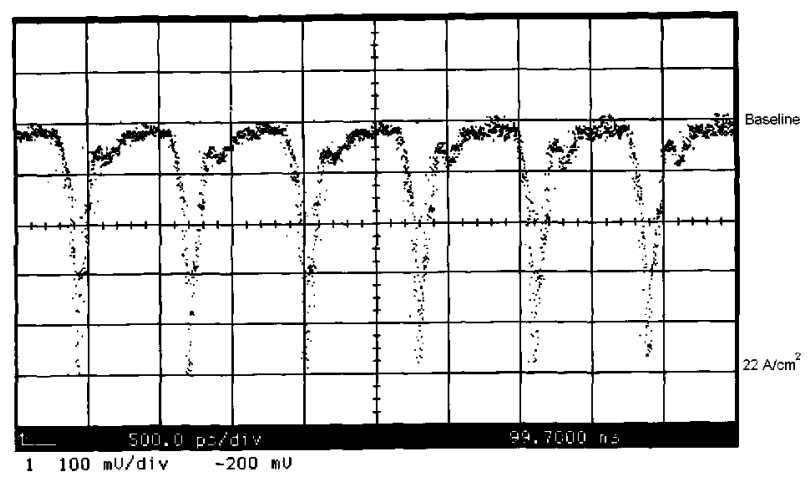

Figure 3: Experimental data showing the current trace of the MPG microbunches.

Figure 4 shows a MPG experiment at S-band (2.85 $\mathrm{GHz}$ ). The micrometer that is vertical in Figure 4 is used to adjust the current. The diagonal micrometer is used to tune the $\mathrm{TE}_{101}$ cavity. The macropulse operation is shown in Figure 5 where the top trace is the RF power in the cavity and the bottom trace is the transmitted macropulse beam current, both on a $0.5 \mu \mathrm{s} / \mathrm{div}$. time scale. The RF 
power is about $400 \mathrm{~kW}$ and the macropulse current is about $18 \mathrm{~A}$. The corresponding micropulse or bunch current is $360 \mathrm{~A}$. The macropulse current density is about 10 $\mathrm{A} / \mathrm{cm}^{2}$ and the corresponding micropulse or bunch current density is $200 \mathrm{~A} / \mathrm{cm}^{2}$. The S-band MPG has been operating at a repetition rate of $50-300 \mathrm{~Hz}$ with a $2.25 \mu \mathrm{s}$ macropulse and $6.3 \mathrm{nC}$ per microbunch. There are 6,412 microbunches or $40.5 \mu \mathrm{C}$ per macropulse. Note also the clean current trace over the full RF pulse length. The Sband work is presently being extended to give a $4.5 \mu \mathrm{s}$ pulse at a frequency of $2.995 \mathrm{GHz}$.

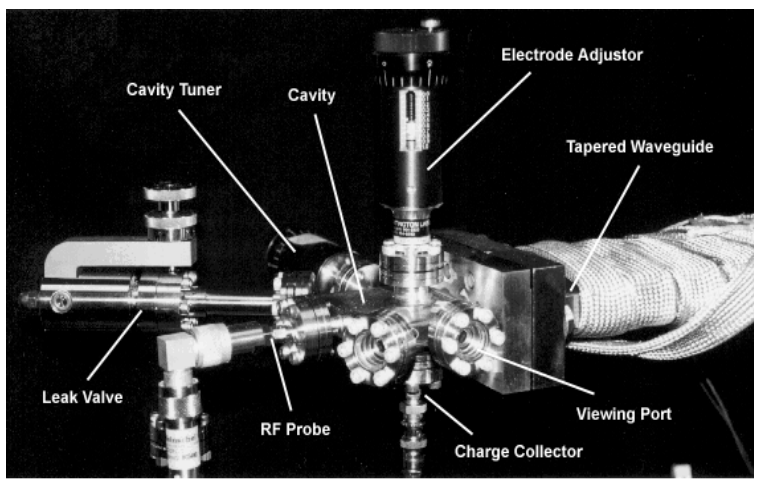

Figure 4: Close-up of the S-band experiment showing the tapered waveguide and the MPG. RF power is fed to the cavity via the tapered waveguide (wrapped in heating tape) from the right. The micrometer on top provide adjustability to the electrode. A charge collector is attached to the coaxial feed-through at the bottom. A port with an RF probe and one with a leak valve are on the left. The micrometer in the back is for cavity tuning.

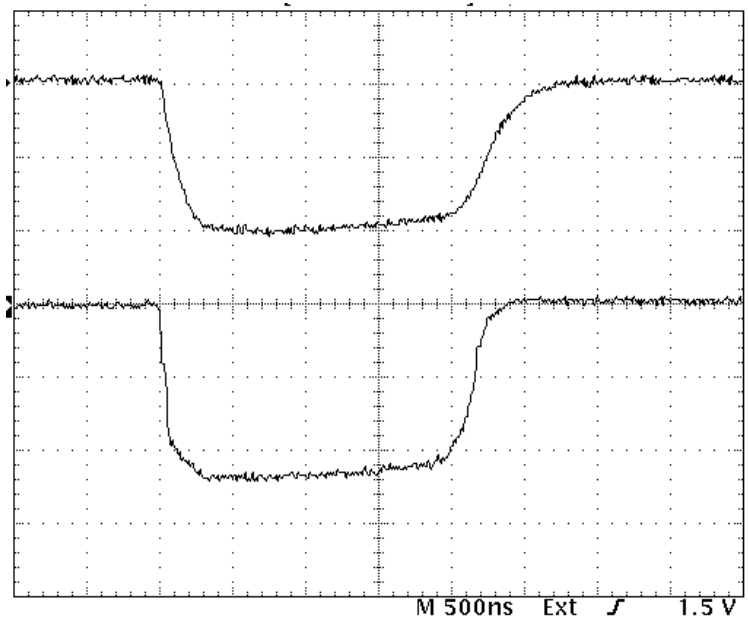

Figure 5: Oscilloscope traces of macropulse operation of the S-band experiment. Top: The RF power in the cavity. Bottom: The transmitted beam current macro pulse.

\section{SUMMARY}

In the last several years, considerable progress has been made in advancing cathode sources of electrons. Extensive work has been done on the field emission source and prototype microwave tubes are being fabricated using field emission arrays. Field emission arrays hold the promise of direct modulation of emission, but this feature has yet to be demonstrated in a practical device. High current densities have been achieved in electron emission from ferroelectrics. In this case, the physics of the emission process is not completely understood on a materials level. These sources show potential for use in high current source applications such as high power microwave tubes. Further work is required to study the useful life of the sources especially at high current, long pulse and high repetition rate. Like field emission arrays, direct modulation of emission is difficult which due to the high capacitance.

An extensive amount of work has been done with photoemitters on the production of single pulses and on the generation of a short train of pulses. The main application has been as injectors for accelerators. Their use in RF generation is at present limited to relatively short duration pulses. Photocathodes are complementary to the MPG device since they can provide a flexible assortment of pulses.

Finally, we have described the MPG, a device which uses secondary emission in an RF cavity to produce a train of picosecond long electron pulses. In contrast to photoemission sources the MPG can easily produce multimicrosecond long macropulses. When driven with a klystron, it can form a very compact and high gain RF system. The fact that the MPG produces prebunched pulses, and doing so before post-accceleration, makes it extremely attractive for use in many applications including RF generation, radiography, radiation therapy, radiation sterilization, and in injector for high-energy accelerators. Its proven long lifetime is a big advantage.

Research and development of new electron emitters has advanced substantially in the last several years. However, these novel emission schemes have not yet received widespread use. Additional research and development is still required to provide improved electron gun solutions for practical applications.

The electron emission schemes presented in this paper are described in more detail in reference [51].

\section{ACKNOWLEDGMENTS}

The authors are grateful to John Nation, Cha-Mei Tang and Triveni Srinivasan-Rao for providing much of the information on ferroelectric emission, field emission and photoemission. 


\section{REFERENCES}

1. J. L. Adamski et al., IEEE Trans. Nucl. Sci. NS-32 (5), 3397 (1985).

2. T. F. Godlove and P. Sprangle, Particle Accelerators, 34, 169, (1990).

3. H. Braun et al., CLIC Note 364, The CLIC RF Power Source, (1998).

4. H. Braun et al., CLIC Note 367 Proc. EPAC 98, CERN/PS 98-014(LP) .

5. E. G. Zaidman and M. A. Kodis, IEEE Trans. El. Dev., ED-38, p. 2221-2228 (1991).

6. W. A. Mackie, R. L. Hartman and Paul R. Davis, Applied Surface Science 67, p. 29-35 (1993).

7. W. A. Mackie, R. L. Hartman, M. A. Anderson and P. R. Davis, J. Vac. Sci. Technol. B 12, p. $722-726$ (1994).

8. L. Parameswaran et al., submitted to Proc. of Vacuum Electronics Review, San Diego, May (1997).

9. C. O. Bozler et al., J. Vac. Sci. Technol. B 12, p. 629 (1994).

10. C. A. Spindt et al., J. Vac. Sci. Technol. B 14, p. 1986 (1996).

11. C. A. Brau, presented at 9th Interntional Free Electron Laser Conf., Beijing, China, August, 1997.

12. C. A. Brau, presented at Gargnano Electron Beam Workshop, 1997.

13. C. M. Tang, M. Goldstein, T. A. Swyden and J. E. Walsh, Nucl. Instrum. and Methods A 358, pp. 7-10 (1995).

14. Y. Liu and Y. Y. Lau, J. Vac. Sci. Technol. B 14, p.2126-2129 (1996).

15. C. M. Tang, 1995 Particle Accelerator Conf., Dallas, TX, p. 70-74 (1995).

16. C. M. Tang and T. A. Swyden, Society for Inform. Display Intl. Sym. Digest of Tech. papers, Vol. XXVIII., p. 115-118 (1997).

17. H. Makishima et al., Technical Digest of IVMC'97 Kyongju, Korea, p. 194-199 (1997).

18. H. Imura et al., Technical Digest IEDM, Intl. Electron Devices Meeting, Wash. D.C., Dec. 7-10, 1997 p.721-724.

19. H. Takemura et al., Technical Digest IEDM, Intl. Electron Devices Meeting, Wash. D.C., Dec. 7-10, 1997 p.709-712.

20. S. G. Bandy et al., 1997 IEEE Intl. Conf. on Plasma Science, IEEE Catalog No. 97CH36085, May 19-22, p. 127 (1997).

21. K. L. Jensen, J. E. Yater, E. G. Zaidman, M. A. Kodis and A. Shih, Tech. Digest IVMC'97, Kyongju, Korea, p.186-189 (1997).

22. D. Palmer et al., J. Vac. Sci. Technol. B 13, p. 576-579 (1995).

23. R. K. Parker, K. L. Jensen and R. H. Abrams, Technical Digest IVMC'97, Kyongju, Korea, p. $92-97$ (1997).

24. C. A. Spindt, C. E. Holland, P. R. Schwoebel and I. Brodie, J. of Vac. Sci. Technol. B 14, p. 1986-1989 (1996).

25. C. A. Spindt, C. E. Holland, P. R. Schwoebel and I. Brodie, Technical Digest of IVMC'97, Kyongju, Korea, p. 200-207 (1997).

26. J. P. Calame, H. F. Gray and J. L. Shaw, J. Appl. Phys. 73, p. 1485-1504 (1993).

27. P. Schoessow et al., Proc. of the 2nd European Particle Accel. Conf., p. 606 (1990).

28. K. Batchelor, et al., Nucl. Instr. and Meth. in Phy. Res. A318, p. 372 (1992).

29. S. C. Hartman et al., presented at 1993 Part. Accel. Conf., IEEE Cat. 93CH3279-1, p. 561 (1993).

30. I. Ben-Zvi, presented at 1993 Part. Accel. Conf., IEEE Cat 93CH3279-1, p. 2962 (1993).

31. I. S. Lehrman, I. A. Birnbaum, M. Cole, R. L. Heuer, E. Sheedy, Part. Accel. Conf., IEEE Cat. 93CH3279-1, p. 3012 (1993).

32. C. Travier et al., Nucl. Instr. and Meth. in Phy. Res. B89, p. 27 (1994).

33. I. Ben-Zvi, Adv. Accel. Concepts, CF 398, 40, AIP Press, NY (1997).

34. R. Bozart et al., CLIC Note 29, PS/RF Note 95-25, CERN, Switzerland.

35. H. Gundel, H. Riege, E.J.N. Wilson, J. Handerek and K. Zioutas, Nuclear Instru. and Methods in Phy. Research A 280 p.1 (1989).
36. T. C. Cavazos, W.L. Wilbanks, C.B. Fleddermann and D.A. Shiffler, Appl. Phys. Lett. 65, p. 2612 (1994).

37. A. Sh. Airapetov, A.K. Krasnykh, I.V. Levshin and A. Yu. Nikitskii, Sov. Tech. Phys. Lett. 35, p.182 (1990).

38. J.D. Ivers, L. Schachter, J.A. Nation, G.S. Kerslick and R. Advani, J. Appl. Phys. 73, p. 2667 (1993).

39. L. Schachter, J.D. Ivers, G.S. Kerslick and J.A. Nation, J. Appl. Phys. 73, p. 8097 (1993).

40. B. Jiang, G. Kirkman and N. Reinhardt, Appl. Phys. Lett. 66, p. 1196 (1995).

41. S.E. Sampayan et al., Nuclear Instruments and Methods in Physics Research A 340, p. 90 (1994).

42. M. Okuyama, J. Asano and Y. Hamakawa, Jpn. J. Appl. Phys. 33, p. 5506 (1994).

43. J.D. Ivers et al., submitted for publication to IEEE Trans. on Plasma Science (1998).

44. F. Mako and W. Peter, Part. Accel. Conf., IEEE Cat. 93CH3279-1 p. 2702 (1993).

45. A. S. Gilmore, Microwave Tubes (Artech House, Norwood, MA 1986), p. 474.

46. J. R. M. Vaughan, IEEE Trans. Electron Devices 35 (7), p. 1172 (1988)

47. R. Kishek and Y. Y. Lau, Phys. Rev. Lett. 75, 6, p. 1218, (1995).

48. S. Riyopoulos, D. Chernin and D. Dialetis, Phys. Plasmas 2 (8), p. 3194 (1995); also IEEE, Trans. Elec. Devices, 44, p. 489 (1997).

49. F.M. Mako and L.K. Len, to be published in the Proceeding of RF98 Conference (1998).

50. F. Mako, L.K. Len and W. Peter, to be published in the Proceedings of the 8th Workshop on Advanced Accelerator Concepts, 1998.

51. J. A. Nation et al., Proc.of the IEEE, vol. 87, no. 5, May 1999. 